NASA Technical Memorandum 101992

\title{
Satellite Relocation by Tether Deployment
}

Geoffrey A. Landis and Frank J. Hrach

Lewis Research Center

Cleveland, Ohio

April 1989

(HA SA-TH-101992) SAT ELLITE RELOCATION BY TER GER DEPLOTHENT (MASA. LeVis Research Center) $16 \mathrm{p}$ CSCL 22B 


\title{
Satellite Relocation by Tether Deployment
}

Geoffrey A. Landis* and Frank J. Hrach

National Aeronautics and Space Administration

Lewis Research Center

Cleveland, OH 44135

\begin{abstract}
Several new uses of satellite tethers are discussed, including: (1) using tether extension to reposition a satellite in orbit without fuel expenditure by extending a mass on the end of a tether; (2) using a tether for energy storage to power the satellite during eclipse; and (3) using a tether for eccentricity pumping to correct perturbations in the orbit and as a means of adding energy to the orbit for boosting and orbital transfer.
\end{abstract}

\section{Orbital Repositioning of a Satellite}

\section{Introduction}

One of the limitations of the working lifetime of a satellite is the expenditure of fuel required for changing and maintaining the orbit. In geosynchronous orbit, the orbit is subject to perturbations, primarily due to the gravitational effects of the sun and the moon. Fuel is also required for relocating a satellite, for example, if a synchronous satellite positioned over Indonesia is desired to be relocated over South America.

A tether is a long, flexible cable which connects one part of a satellite with another. Tethers have recently been well covered in the aerospace literature [ref. 1-5]. In the equilibrium configuration, as shown in figure 1, the tether is oriented radially outward, with a tension on the tether due to the gravitational gradient (or "tidal") force.

*National Research Council-NASA Research Associate. 
Mass of the cable is an important figure. A figure of merit for material strength is the critical length $L_{c}$, the length of untapered cable that could be suspended in a 1-g gravitational field. One proposed cable material is Kevlar, for one common type of which the critical length is $250 \mathrm{~km}$ [6]. The effective acceleration due to the gravity gradient a distance $x$ from the center of mass $(\mathrm{CM})$ is, to first order:

$$
a_{e j \rho}=3 g_{o} r_{e}^{2} x / r_{o}^{3}
$$

where $g_{o}$ is the gravity at the Earth's surface, $r_{o}$ is the orbital radius and $r_{e}$ is the radius of the earth. At geosynchronous Earth orbit (GEO), $3 \mathrm{r}_{e}^{2} / \mathrm{r}_{o}^{3}=1.6 \cdot 10^{-6} \mathrm{~km}^{-1}$. The minimum mass $\mathrm{m}_{t}$ of untapered cable required to support an end mass $\mathrm{m}_{o}$ is thus to first order (for $x$ in $\mathrm{km}$ ):

$$
m_{t}(G E O)=1.6 \cdot 10^{-6} m_{o} x^{2} / L_{c}
$$

Thus, in GEO a $1000 \mathrm{~km}$ long tether can easily be made much less massive than the satellite it supports.

Most analyses of tether orbits assume that the center of mass of a tethered satellite system remains in the original orbit $[1,2]$; i.e., that the angular velocity of the tethered satellite does not change as the tether is extended or retracted. We note that this is true only to the first order approximation in tether length. Briefly, the mass that extends outward experiences an increase in centrifugal force that increases linearly with distance, but the mass that extends inward experiences an increase in gravity that increases faster than linearly. Thus, the center of mass of the orbit is pulled inward, and to conserve angular momentum, the angular velocity of the orbit increases. 


\section{Mathematical Analysis}

In the following discussion we assume a tether of negligible mass in circular orbit. The extension of the analysis to tethers of non-negligible mass is straightforward.

Consider a satellite of mass $m_{i}$ consisting of two pieces of mass $m_{1}=m_{2}=m_{i} / 2$ connected by a tether. The initial orbit is assumed to be circular, with an angular velocity $\omega_{o}$ and an initial orbital radius (measured from the Earth's center) $\mathbf{r}_{o}$. With the tether at initial length zero, the orbit has initial angular momentum

$$
L_{i}=m_{i} \omega_{o} r_{o}^{2}
$$

Force balance (outward centrifugal force equals inward gravitational force) requires $\omega_{o}^{2} r_{o}^{3}=\mathrm{GM}$, where GM is the gravitational constant times the mass of the Earth. Now assume that the tether is extended to length $x$ in each direction from the CM, as shown in figure 1. The total length is $2 x$. Note that energy is not conserved, since in deploying a tether work is done by the effective tidal force. Angular momentum is still conserved,

$$
L=m_{1} \omega r_{1}^{2}+m_{2} \omega r_{2}^{2}
$$

where $\mathrm{r}_{c m}$ is the orbital radius of the CM, and $\mathrm{r}_{1}=\mathrm{r}_{c m}-x$ and $\mathrm{r}_{2}=\mathrm{r}_{c m}+x$. The system must also have a force balance, which implies (for a massless tether) that the inward tension on the low end of the tether equals the outward tension on the high end of the tether,

$$
\omega^{2}\left(r_{1}+r_{2}\right)=G M \cdot\left(r_{1}^{-2}+r_{2}^{-2}\right)
$$

If we expand this to second order in $x$, then set equation (3) equal to equation (4) to solve equations (5) for $\omega$ and $r_{c m}$ as a function of tether extension $x$, we find 


$$
r_{c m}=r_{o}-5 \frac{x^{2}}{r_{o}}
$$

and the orbital period $\mathrm{P}$ is

$$
P=P_{o}\left[1-9\left(\frac{x}{r_{o}}\right)^{2}\right]
$$

The result is that the orbital period decreases as the tether length is extended. For example, a GEO satellite consisting of two equal masses on a $1000 \mathrm{~km}$ long tether will have a period faster than that of an untethered satellite by $0.44^{\circ}$ per day.

Inclusion of higher order terms results in an increase in the effect.

If the two masses are allowed to differ, the orbital period change is proportional to $m_{1} m_{2} /\left(m_{1}+m_{2}\right)$, which is maximum when the two masses are equal.

\section{Satellite Relocation}

We propose to use this effect to relocate a satellite without expenditure of fuel. Consider a satellite in orbit which consists of two masses connected by a tether with a motorized spool which allows it to be pulled out or in. Preferably the spool contains a motor/generator, such that the energy used pulling the tether in can be regained (except for friction losses) when the tether is let out. It is also important that the spool have a controller that can be programmed to damp lateral oscillations in the tether during the reeling process.

The spool motor can be powered by the satellite solar array, which does not expend of fuel.

Pulling the tether in will result in a longer orbital period for the satellite, and thus drift the satellite backward (west) in longitude; while letting the tether out will result in a shorter orbital period, and thus drift the satellite forward (east). This is shown schematically in figure 2 . 
Maximum relocation capability is achieved when the masses on both ends of the tether are equal. The countermass could be the spent booster used to boost the satellite up from low Earth orbit or the apogee kick motor. Alternatively, the countermass could be systems which do not require to be located in close proximity to the lower part of the satellite, or could be an entirely separate satellite system. Finally, if the tether is sufficiently long, no counterweight would be needed, with the mass of the tether itself providing this function. In this case a single tether could extend either inward or outward.

In the normal operating position, the tether would be extended to a length $1 / \sqrt{2}$ of full length (about 0.707). If the tether is $1000 \mathrm{~km}$ long, this allows the satellite to be moved forward as fast as $0.22 \%$ day by fully extending the tether, or to lag backward up to $0.22^{\circ}$ /day by fully retracting it. Thus, relocation by a few degrees in longitude could be accomplished in a week or so, and the worst possible case, $180^{\circ}$ relocation, in two years.

The preceeding analysis has assumed equilibrium conditions, i.e., that the orbit remains circular during the extension and deployment of the tether. This assumption is true only if the tether is deployed or retracted over a time greater than an orbital period. Faster deployment will result in dynamic changes to the orbital eccentricity. This is discussed in more detail in section 3 .

\section{Energy Storage}

Note that the tether need not be an otherwise inactive part of the satellite. Since energy is gained in deploying the tether out and used in pulling the tether in, it could be used for energy storage. An optimal design for a geosynchronous satellite might well use this energy storage in place of batteries to power the satellite electrical systems during the eclipse period (which lasts about 1.1 hours per day for a period of several days around the equinox), when no solar energy is available to power the solar cells. The small orbital change produced by this could be easily corrected by reeling 
the tether in to compensate during the sunlit portion of the orbit. By integrating equation 1 , the total energy available is:

$$
E=1.5 m g_{o} L^{2} r_{e}^{2} / r_{o}^{3}
$$

For $\mathrm{E}$ the energy in watt-hrs, $\mathrm{L}$ the half-length in $\mathrm{km}$, and $\mathrm{m}$ the satellite mass in $\mathrm{kg}$, in GEO the specific energy is $0.55 \mathrm{~W}$-hrs per kilogram of satellite mass. From equation 2 , the maximum energy that can be stored per kilogram tether mass is

$$
\frac{E}{m_{t}}=0.5 g_{o} L_{c}
$$

independent of orbital radius. For a kevlar cable, this is about $350 \mathrm{~W}-\mathrm{hr} / \mathrm{kg}$. Even including a large safety margin, this compares favorably to the $\sim 25-35 \mathrm{~W}-\mathrm{hrs} / \mathrm{kg}$ produced by current technology $\mathrm{Ni}-\mathrm{H}$ batteries [7].

The tether may have additional uses on the satellite as well. In addition to correction of small perturbations in the orbital period (e.g., due to lunar and solar perturbations) by adjusting the tether length, modulation of the length in phase with the orbital period could be used to adjust changes in the orbital eccentricity as discussed in the following section. The eccentricity of an orbit can be changed without changing the angular momentum, by adding orbital energy by modulating the length of a tethered satellite. This process can be used for orbit-to-orbit propulsion. Another possible use for the tether is as an element of the initial boost of the satellite into position, by the well-researched process of tether boost $[1,2]$, as shown in figure 3 .

\section{Eccentricity Pumping}

\section{Introduction}

It has previously been shown that the orbital eccentricity of a tethered spacecraft can be changed by modulating the tether length $[8,9]$. In this paper the use of 
this effect as a means for orbital propulsion that does not require expenditure of reaction mass is discussed. In the process energy is added to the orbit (from a power source on board the spacecraft), while the orbital angular momentum is constant. Particular applications discussed are injection of a spacecraft into an escape orbit from an initially circular orbit, and use of the process for transfer orbits, e.g., LEO to GEO.

The method is similar to oblateness pumping [2], but does not require deviation from spherical symmetry and can be done from an equatorial orbit.

The reverse process, circularizing an eccentric orbit by removal of energy by a viscoelastic tether, has been discussed in detail by Columbo et al. [10].

We also note that, contrary to expectations, the eccentricity pumping process is most effective when the orbit is nearly circular. Although the amount of energy available per orbit decreases as the orbit becomes nearly circular, the sensitivity of the eccentricity to small changes in energy increases as $1 / \epsilon$, and this factor dominates over the decrease in energy. For a perfectly circular orbit, higher order terms contribute as well.

The method is straightforward. A mass is deployed away from the spacecraft on the end of a tether. The stable configuration is with the tether oriented radially from the central body. The tether is mounted on a reel with a motor which can pull it in or let it out. The method of orbit pumping consists of pulling the tether in at perigee (more generally, periapsis) and letting the tether out at apogee. Since gravitational gradient (tidal) forces are to first order proportional to the inverse radial distance cubed, more mechanical work is done against the tidal force in pulling the tether in than is returned when the tether is let back out. Thus, energy is added to the orbit. Since the orbital angular momentum is unchanged, the eccentricity $\epsilon$ of the orbit increases.

Eccentricity pumping can only be done if the initial orbit is high enough that the minimum perigee does not impact the primary. For pumping from an initial circular 
orbit at distance a to escape, recognizing that at perigee of the escape parabola $\mathbf{v}$ $=\sqrt{2}$ times orbital velocity, this implies that $a_{o} \geq 2 r_{e}$. In general, the minimum perigee will also desirably be high enough not to intersect the atmosphere; $r_{e(e f s e c t i v e)}$ $=\mathrm{r}_{e}+\sim 200 \mathrm{~km}$. This corresponds to an initial orbital radius of $\sim 13,150 \mathrm{~km}$, or a minimum initial orbital altitude of $6,575 \mathrm{~km}$.

Figure 4 shows the pumping method in schematic.

\section{Mathematical Analysis}

We assume here that the tether itself is of negligible mass. The case of a massive tether can be straightforwardly calculated by integration over the mass distribution. We also assume that the masses on each end of the tether are equal, the case which for fixed tether length maximizes the effect. Again, extrapolation to unequal masses is straightforward.

The gravity gradient for an orbit a distance $x$ from the center of mass is (from equation (1):

$$
F=3 \frac{r_{e}^{2} x}{r_{o}^{3}} m g_{o}
$$

where $r_{e}$ is the radius of the Earth, and $r_{o}$ the distance of the orbit from the center of the planet. Now assume equal masses $\mathrm{m} / 2$ extended on the ends of a tether of full length $\mathrm{d}$ (i.e., half-length $x$ ). The mechanical energy stored in the tether is thus

$$
E=\frac{3}{8} m g_{o} \frac{r_{e}^{2}}{r_{o}^{2}} d^{2}
$$

Now assume the orbit is elliptical. The orbital energy is

$$
E=-m g_{o} \frac{r_{e}^{2}}{2 a}
$$

where $a$ is the semimajor axis of the orbit. From conservation of angular momentum, the semimajor axis of the ellipse must be related to the eccentricity $\epsilon$ by

$$
a=\frac{a_{o}}{1-\epsilon^{2}}
$$


where $a_{o}$ is the semimajor axis of a circular orbit of the same angular momentum and $\epsilon$ is the eccentricity. Thus,

$$
E=-m g_{o} \frac{r_{e}^{2}\left(1-\epsilon^{2}\right)}{2 a_{o}}
$$

The apogee and perigee altitudes are:

$$
r_{\text {apogee }}=a(1+\epsilon)=\frac{a_{o}}{(1-\epsilon)}
$$

and

$$
r_{\text {perigee }}=a(1-\epsilon)=\frac{a_{o}}{(1+\epsilon)}
$$

Thus, the amount of energy required to retract the tether at perigee minus the amount recovered in extending the tether at perigee is:

$$
\frac{\Delta E}{\text { orbit }}=\frac{3}{8} m g_{o} d^{2} \frac{r_{e}^{2}}{a_{o}^{3}}\left[(1+\epsilon)^{3}-(1-\epsilon)^{3}\right]=\frac{3}{8} m g_{o} d^{2} \frac{r_{e}^{2}}{a_{o}^{3}} \epsilon\left(6+\epsilon^{2}\right)
$$

In the real case, the tether length $d$ will not reeled in all the way to zero length. An effective value of $\mathrm{d}$ can be used, $d_{e f f}^{2}=d_{\max }^{2}-d_{\min }^{2}$.

By solving (5) for $\epsilon$ and differentiating, the sensitivity of $\epsilon$ to changes in energy is

$$
\frac{d \epsilon}{d E}=\frac{a_{o}}{m g_{o} r_{e}^{2} \epsilon}
$$

The orbital period is

$$
\tau=\frac{2 \pi}{r_{e} \sqrt{g}_{o}}\left[\frac{a_{o}}{\left(1-\epsilon^{2}\right)}\right]^{\frac{3}{2}}
$$

or, expressed in terms of the orbital period of the initial circular orbit,

$$
\tau=\tau_{o}\left(1-\epsilon^{2}\right)^{-\frac{3}{2}}
$$

By dividing equation (8) by equation (11) we calculate the average power expended,

$$
\frac{d E}{d t}=\frac{3}{8} m g_{o} \frac{r_{e}^{2}}{a_{o}^{3} \tau_{o}} d^{2} \epsilon\left(6+\epsilon^{2}\right)\left(1-\epsilon^{2}\right)
$$

The function of $\epsilon$ has a maximum of 2.45 at $\epsilon=0.6$. In practical units, this is an average specific power of

$$
\overline{\mathrm{P}}=(280 w / k g) \mathrm{a}_{o}^{-9 / 2} L^{2} \epsilon\left(2.45+0.41 \epsilon^{2}\right)\left(1-\epsilon^{2}\right)
$$


where $a_{o}$ is the initial semimajor axis in multiples of the earth radius $r_{e}$ and $L$ is the tether length in thousands of kilometers. Note that the function of $\epsilon$ has been normalized to a maximum value of 1 . Since for most applications $d$ will be $<1000$ $\mathrm{km}$ and $\mathrm{a}_{o}$ will be $>\sim 1.5 \mathrm{r}_{e}$, this specific power is well within achievable levels. The peak power levels required will be higher, and depend on how fast the tether is reeled in.

Then the rate of change of eccentricity is

$$
\frac{d \epsilon}{d t}=\frac{d \epsilon}{d E} \frac{d E}{d t}=\frac{3}{8 \tau_{o}} \frac{d^{2}}{a_{o}^{2}}\left(6+\epsilon^{2}\right)\left(1-\epsilon^{2}\right)^{\frac{3}{2}}
$$

Figure 5 shows the rate plotted against eccentricity. As the eccentricity approaches 1 (i.e., the orbit approaches a parabolic escape orbit), the rate drops to zero.

To find the time required to reach a given eccentricity, this expression is inverted and integrated. The integral can be done exactly,

$$
t(\epsilon)=\frac{8}{3} \tau_{o} \frac{a_{o}^{2}}{d^{2}} \frac{1}{7 \sqrt{42}} \tan ^{-1}\left[\frac{7 \epsilon}{\sqrt{42\left(1-\epsilon^{2}\right)}}\right]+\frac{\epsilon}{7 \sqrt{1-\epsilon^{2}}}
$$

Figure 6 shows the time to reach a given eccentricity plotted. Escape is approached asymptotically. It is also of interest to look at the time required to reach a given apogee altitude; this is shown in figure 7 .

As an example, consider the case where eccentricity pumping is used to move from LEO to geosynchronous transfer orbit. $a_{G E O}=6.63 \mathrm{r}_{e}$, so $a_{L E O-G E O}=3.82 \mathrm{r}_{e}$, and $\epsilon_{L E O-G E O}=0.738$. The minimum perigee requirement leads to a minimum initial orbital radius of $\mathrm{a}_{o}=1.74 \mathrm{r}_{e}$, i.e., initial orbital altitude $4700 \mathrm{~km}$ and initial orbital period $\tau_{o} \sim 200 \mathrm{~min}$. From figure 6 , the time needed is $0.43 \tau_{o}\left(\frac{a_{a}}{d}\right)^{2}$.

For a $500 \mathrm{~km}$ tether length, $\left(\frac{a_{a}}{d}\right)^{2} \sim 500$, and the orbital pumping process takes 725 hours, or about 31 days.

A efficient technique for the apogee kick would be to continue eccentricity pumping until the apogee is well past GEO, perform the apogee kick, then use eccentricity pumping in reverse to circularize the orbit. The amount of velocity change 
$\Delta \mathrm{V}$ required to give the orbit sufficient angular momentum to attain circular orbit at GEO is inversely proportional to the distance. Thus, in theory, the $\Delta \mathrm{V}$ needed for apogee kick could be made arbitrarily low by pumping the apogee to a high enough initial value, although this would take a long time.

The decrease in eccentricity rate with increasing eccentricity is due to several factors, one of them being that the energy transfer per orbit is proportional to the square of the tether length over the orbital semimajor axis. As the eccentricity, and thus the semimajor axis, increases, the rate decreases. Since the tether is retracted at perigee, the effect can be eliminated by increasing the tether length as the apogee distance increases. (As tether length increases, this will require initiating the retraction slightly before perigee.) Note that since the total stress due to tidal force goes as $\mathrm{d}^{2} / \mathrm{r}^{3}$, the maximum stress at apogee decreases despite the increased tether length. The requirement for a minimum initial altitude comes from the necessity that the minimum perigee of the orbit not intercept the atmosphere. This requirement can be alleviated if the pumping maneuver is combined with an incremental $\Delta \mathrm{V}$ at each apogee to increase the angular momentum just sufficiently to keep the perigee from decreasing.

\section{Conclusions}

A new method has been described for a satellite in orbit to be repositioned in orbital longitude by use of a tether connecting the active satellite with an inert mass, such as the expended booster. In addition to allowing repositioning, the system also allows correction of small changes in orbital period and eccentricity.

The tether can be used for energy storage, for example, to power a satellite during eclipse.

The tether can be used for changing the orbital eccentricity of the satellite without changing the angular momentum. This process can be used to correct eccentricity 
perturbations. Since the process adds energy to the orbit, it can also be used as an orbital boost method, or for orbit to orbit transfer.

\section{References}

1. I. Berkey and P.A. Penzo, "Tether Propulsion," NASA/AIAA/PSN International Conference on Tethers in Space, 17-19 Sept. 1986, Arlington VA; reprinted in Aerospace America July 1986.

2. W.A. Baracat and C.L. Butner, Tethers in Space Handbook, printed by NASA Office of Space Flight, N88-14123, August 1986.

3. D.A. Arnold, "The Behavior of Long Tethers in Space," J. Astronautical Sci., Vol. 35, No. 1, 3-18 (1987).

4. W.A. Baracat, ed., Applications of Tethers in Space, NASA Conference Publication 2422, 1986.

5. P.M. Bainum et al. (ed), Advances in Astronautical Science Volume 62, Tethers in Space, AAS, San Diego (1987).

6. E. Levy, Jr., "U.S. Air Force Design Nickel-Hydrogen Cells: Flight Status and Recent Improvements," paper 869348, Proc. 21st Intersociety Energy Conversion Engineering Conference, 1537-1540 (1986).

7. A.S. Brown, "Spreading Spectrum of Reinforcing Fibers, Aerospace America, Jan. 1989 , p. 16.

8. W.A. Baracat and C.L. Butner, Op. cit., p. 3-53.

9. G. von Tiesenhausen, "The Roles of Tethers on Space Station," NASA TM 86519 , Oct. 1985.

10. G. Columbo, M. Grossi, D. Arnold, and M. Martinez-Sanchez, Orbital Transfer and Release of Tethered Payloads, Final Report 1 Sept. 1979-28 February 1983, Report Number N83-25751, Mar. 1983. 


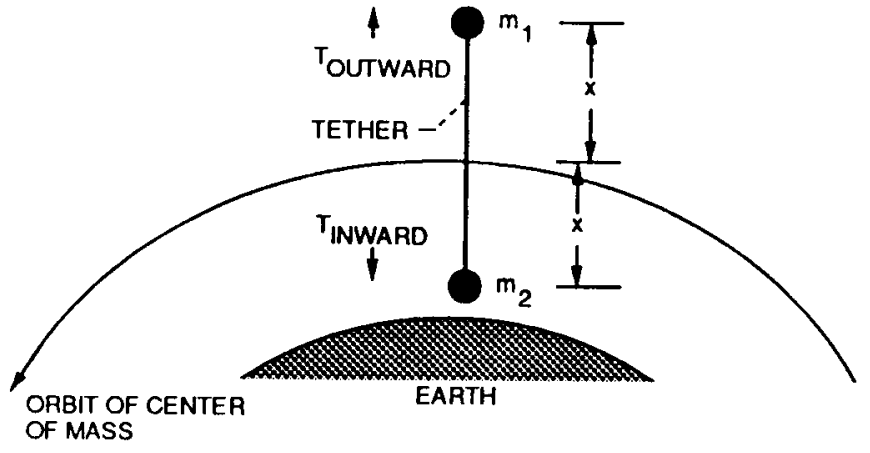

Figure 1. - Tether orbit and definitions.

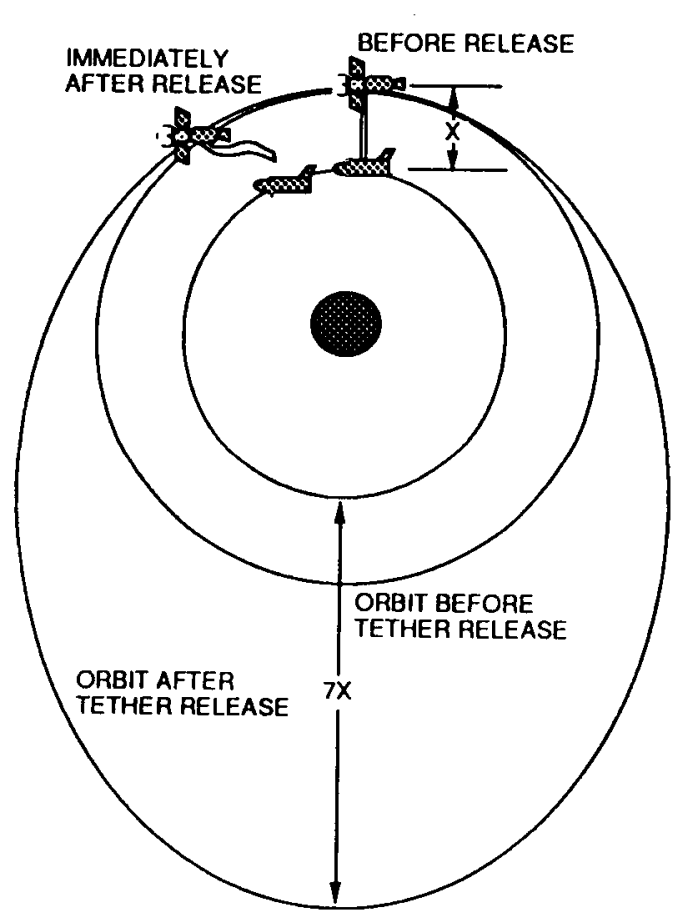

Figure 3. - Use of a tether to boost spacecraft allitude

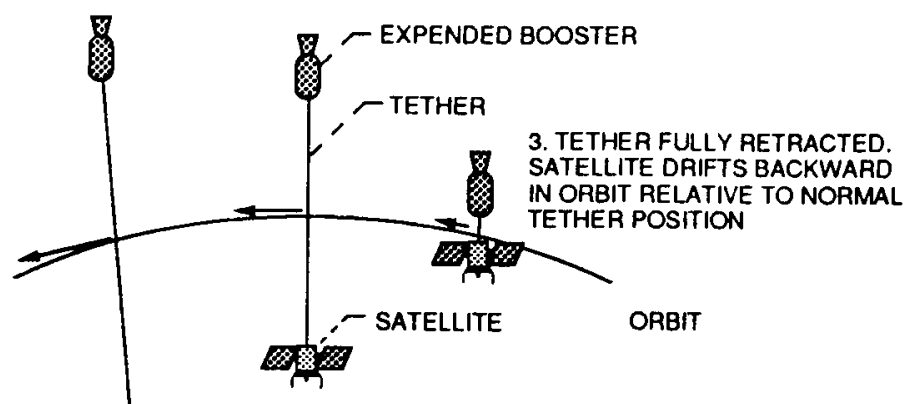

2. NORMAL TETHER POSITION

1. TETHER FULLY EXTENDED.

SATELLITE DRIFTS FORWARD IN

ORBIT RELATIVE TO NORMAL

POSITION

EARTH

Flgure 2. - Satellite relocation by extension and retraction of a tether.

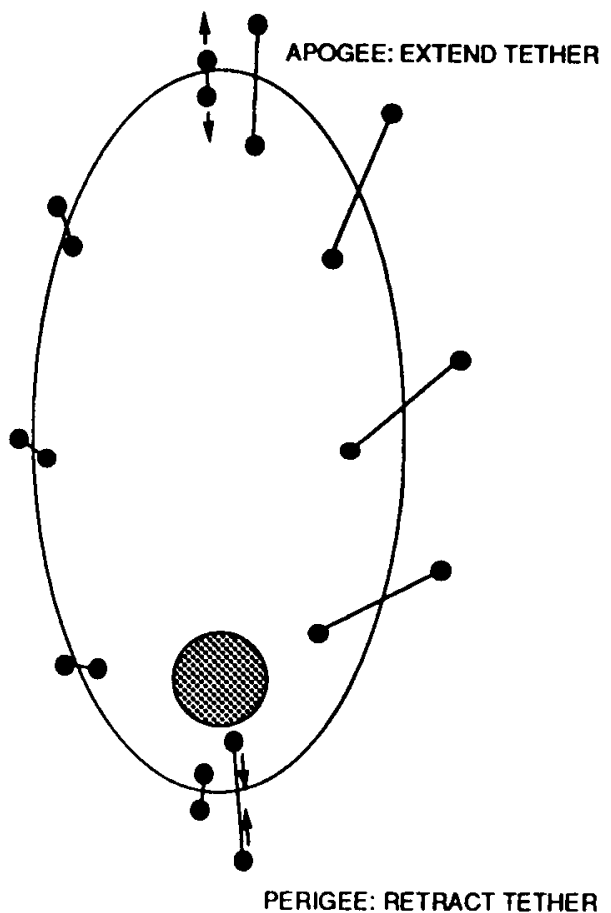

Figure 4. - Eccentricity pumping (schematic). Tether is retracted at perigee of orbit, extended at apogee. 


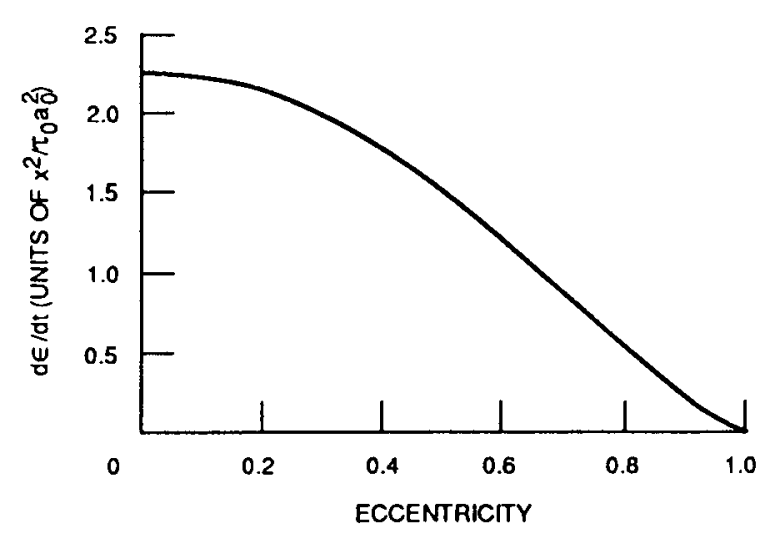

Figure 5. - Rate of eccentricity change vs. eccentricity.

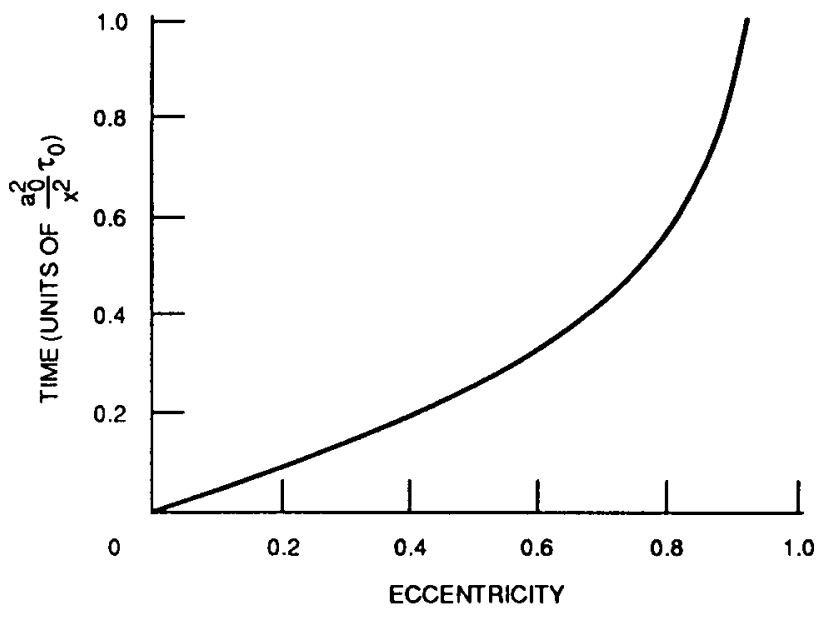

Figure 6. - Time required to attain a given eccentricity.

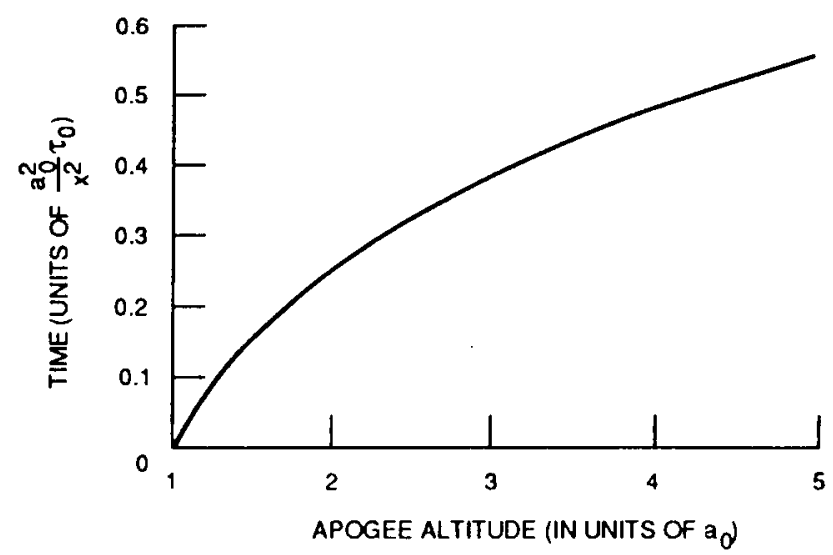

Figure 7. Time required to reach a given apogee altitude. 


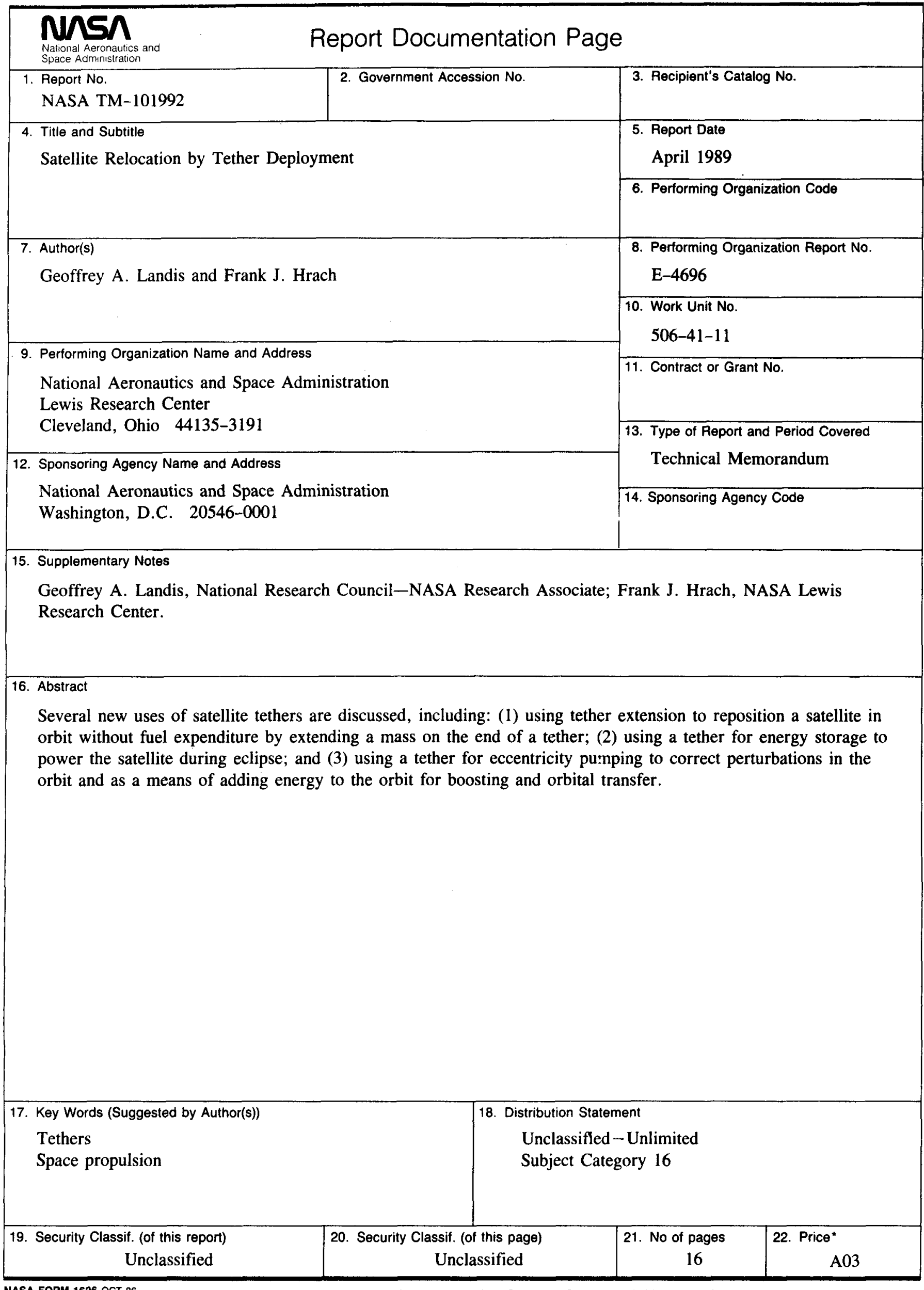

